DOI: $10.4274 /$ tjps.12599

\title{
OPTIMIZATION OF THIAZOLIDONE SCAFFOLDS USING POCKET MODELLING FOR DEVELOPMENT OF POTENTIAL SECRETARY SYSTEM INHIBITORS OF MYCOBACTERIUM TUBERCULOSIS
}

S. V. Khare ${ }^{1}$, S.P Choudhari ${ }^{3}$, S.P.Phalle ${ }^{1}$, S. S. Kumbhar ${ }^{1}$, P. B. Choudhari ${ }^{1 *}$,

S. R. Masal ${ }^{1}$, A. K. Patil ${ }^{1}$, R. P. Dhavale ${ }^{2}$, D. A. Bhagwat ${ }^{2}$, A. M. Kadam ${ }^{4}$

1. Computational Chemistry Research Lab, Department of Pharmaceutical Chemistry, Bharati Vidyapeeth College of Pharmacy, Kolhapur.

2. Department of Pharmaceutics, Bharati Vidyapeeth College of Pharmacy, Kolhapur.

3. Department of Pharmaceutical Analysis, Sarojini College of Pharmacy, Rajendra Nagar, Kolhapur.

4. Department of Pharmaceutics Shree Santkrupa College of Pharmacy, Ghogaon

Author for correspondence

Dr. Prafulla B Choudhari

Department of Pharmaceutical Chemistry

Bharati Vidyapeeth College of Pharmacy,

Near Chitraganeri, Morewadi, Kolhapur, Maharashtra, India

416013

praffula12@gmail.com, prafulla.choudhari@bharatividyapeeth.edu 


\begin{abstract}
:
Objectives: Mycobacterium tuberculosis is a causative organism of tuberculosis, which is most lethal disease after cancer in a current decade. The development of multidrug and broadly drugresistant strains making the tuberculosis problem more and more critical. In last 40 years, only one molecule is added to the treatment regimen. Generally, drug design and development programs are targeted proteins whose function is known to be essential to the bacterial cell. Mycobacterium. tuberculosis possesses specialized protein export systems like SecA2 export pathway and ESX pathways.
\end{abstract}

Material and methods: In the present communication, rational development of anti-mycobacterial agent's targeting protein export system has been carried out by integrating the pocket modeling and virtual analysis.

Results: The identified potential 23 lead compounds were synthesized, characterized by physicochemical and spectroscopic methods like IR, NMR spectroscopy and further screened for anti-mycobacterial activity using isoniazid as standard. All the designed compounds have shown profound anti-mycobacterial activity.

Conclusion: In this present communication, we found that Q30,M9, M26, U8, and R26 molecules had significant desirable biological activity and specific interactions with Sec of mycobacteria. Further optimization of these leads is necessary for the development of potential antimycobacterial drug candidate less side effects.

Keywords: Mycobacterium tuberculosis, Sec, ESX, Docking, Anti-mycobacterial, MDR, Pocket Modeling. 


\section{Özet:}

Amaç: Mycobacterium tuberculosis, son on y1lda kanserden sonra en ölümcül hastalık olan tüberkülozun etken bir organizasyondur. Çoklu ilaç ve geniş ilaca dirençli suşların gelişimi, tüberküloz problemini daha da kritik k1lmaktadır. Son 40 yılda, tedavi rejimine sadece bir molekül eklenmiştir. Genel olarak ilaç tasarım ve geliştirme programları, işlevi bakteriyel hücre için gerekli olduğu bilinen hedeflenen proteinlerdir. Mycobacterium tuberculosis, SecA2 ihracat yolu ve ESX yolları gibi özel protein ihracat sistemlerine sahiptir.

Gereç ve yöntemler: Mevcut iletişimde, anti-mikobakteriyel ajanın hedefleyici protein ihracat sisteminin rasyonel gelişimi, cep modellemesi ve sanal analiz ile bütünleştirilerek gerçekleştirilmiştir.

Bulgular: Belirlenen potansiyel 23 kurşun bileşikleri sentezlendi, bunlar IR, NMR spektroskopisi gibi fizikokimyasal ve spektroskopik yöntemler ile karakterize edildi ve standart olarak izoniazid kullanılarak anti-mikobakteriyel aktivite için daha fazla elendi. Tasarlanan tüm bileşikler, derin anti-mikobakteriyel aktivite göstermiştir.

Sonuç: $\mathrm{Bu}$ mevcut iletişimde Q30,M9, M26, U8 ve R26 moleküllerinin önemli biyolojik aktiviteye ve Mikobakterinin Sec ile spesifik etkileşimlere sahip olduğunu bulduk. Potansiyel antimikobakteriyel ilaç adayının daha az yan etkilerinin gelişmesi için bu potansiyellerin daha fazla optimizasyonu gereklidir. 


\section{Introduction:}

Tuberculosis is the air-bone disease caused by infection with Mycobacterium tuberculosis. In current decade, tuberculosis is emerged as a global emergency due to its mortality rate $1-3$ Tuberculosis act as the salient killer in patients suffering from the immunocompromised diseases like AIDS and diabetics. In more than $80 \%$ cases of Acquired immune deficiency syndrome (AIDS) death occurs in the patients due to tuberculosis ${ }^{4-6}$. The generation of Multidrug Resistant Tuberculosis (MDR), extensively drug-resistant tuberculosis (XDR) and Total drug-resistant tuberculosis (TDR) problem of tuberculosis reached its peak ${ }^{8-11}$. Bedaquiline is only new developed and approved the drug for active MDR tuberculosis in last of two decades. Negligence of pharmaceutical scientist and medicinal chemist towards tuberculosis generated this global problem of tuberculosis. A number of hurdles are normally associated with antitubercular drug discovery; one of them is Mycobacterium tuberculosis. Mycobacterium tuberculosis is lipid-rich gram-negative organism having specialized systems, which make it different from other microorganisms ${ }^{12-15}$. Secretory systems are one of the specialized systems present in Mycobacterium tuberculosis, which are key regulators of virulence of MTB. In Mycobacterium tuberculosis three different secretory systems like Secondary translocase pathway (SEC), twin arginine translocation (TAT) and ESX are present in Mycobacterium tuberculosis ${ }^{16-18}$. SEC or secondary translocase Pathway is a major protein export systems present in the mycobacterium, which is accounting for more than $50 \%$ virulence protein exports in the mycobacterium. Secondary translocase pathway (SEC) is conserved protein pathway present in the mycobacterium, which does not have any homologues in the mammalian systems, which is indeed possessing ideal properties to act as potential anti-mycobacterial drug target. SEC pathway is a key enzyme involved in transport of the virulence protein across the cell membrane which in deed spread the 
tuberculosis all over the host body. Inhibition of SEC will be able to block the transport of the virulence protein, which will inhibit spread of the tuberculosis. In recent years, number of researchers are trying for the development of novel antitubercular agents targeting conserved protein targets. Currently number of molecules like equisetin derivatives ${ }^{19}$, thiazolo[4,5d]pyrimidine derivatives ${ }^{20}$ are reported as SEC inhibitors. In this research article, an attempt has been made for optimization of thiazolidine scaffolds as potential SEC inhibitors via integration of pocket and pharmacophore modeling.

\section{Experimental}

\subsection{Selection of Target:}

Mycobacterium tuberculosis is three specialized protein export systems like SEC, TAT, and ESX. Secondary translocase pathway (SEC) is major protein export systems of MTB, which export bulk of virulent protein, which accounts for the spread of disease. SEC systems are not having any homologs in mammalian systems, so inhibition of this SEC will not lead to any toxicity to the human being. Thus due to the critical role of the SEC in the growth and virulence of the MTB, SEC has been selected as preferred biomolecular target than TAT and ESX

\subsection{Pocket modeling of selected protein export systems;}

Pocket modeling of the selected SEC of MTB was carried out using crystal structure of SEC downloaded from the free protein database www.rcsb.org. Downloaded crystal structure of SEC (PDBID 1QNX) was first refined using biopredicta molecule, via removal of water molecules and retaining native hydrogen atoms in the protein structure. Pocket modeling of SEC A is carried out using proviz module of Vlife MDS 4.4. Proviz is integrated property visualization module using which electrostatic and hydrophobic mapping of biomolecules can be carried out. 


\subsection{Design of molecules:}

Pocket modeling of the SEC revealed the binding pocket of SEC is U shaped and highly hydrophobic, keeping in mind complementary structures on thiazole template were designed with structural modification on the aromatic moiety has been carried out. A number of the aromatic benzaldehyde are utilized to generate number of thiazole derivatives. Total $75 \times 75$ benzaldehyde combinations are utilized to generate total 5625 different structures (indicated as R) as shown in table 1 . These 5625 different thiazole derivatives were designed and drawn in 2D geometry using builder module of the Vlife MDS 4.4. 2D structures are converted into the 3D geometry and their coordinates were optimized via energy minimization process via application of Merck molecular force field (MMFF)

\subsection{Docking analysis of synthesized ligands}

Molecular docking was performed to identify potent derivatives with the maximum binding affinity for SEC of MTB amongst the designed set of molecules. Docking analysis was performed in the biopredicta module of the Vlife MDS 4.4 using grip based docking analysis in which protein structure was kept rigid and molecules are kept the flexible conformation so that number of conformation can be achieved. Best 100 confirmation of the each designed molecules were generated. Potent molecules were scrutinized based on binding energy and interaction profile. The proposed synthetic scheme of designed derivatives is shown in Fig. 1.

\subsection{Screening based on the drug-like properties and percentage absorption.}

Designed set of molecules were analyzed for Lipinski parameters like molecular weight, H-bond acceptor, H-bond donor, Rotatable bond, XlogP etc. The physicochemical descriptors calculated with the help of QSAR module of Vlife MDS 4.4. Total polar surface area (TPSA) calculated from 
the web server www.molinspiration.com/cgi-bin/properties by drawing the molecules in drawing area then calculates the TPSA. Percentage absorption was calculated by using the formula, \% Absorption $=109-(0.345 \times$ TPSA $) .{ }^{21}$ 
Table No. 1: Designed set of molecules (Total $75 X 75$ benzaldehyde combinations are utilized to generate total 5625 different structures indicated as $\mathbf{R})$

\begin{tabular}{|c|c|c|c|c|c|c|c|}
\hline Sr. no. & $\mathbf{R}$ & Sr. no. & $\mathbf{R}$ & Sr. no. & $\mathbf{R}$ & Sr. no. & $\mathbf{R}$ \\
\hline 1. & $\mathrm{C}_{6} \mathrm{H}_{5}$ & 2. & 4- $\left(\mathrm{CH}_{3}\right)_{2} \mathrm{~N}-\mathrm{C}_{6} \mathrm{H}_{5}$ & 3. & $4 \mathrm{CH}_{3} \mathrm{CONH}-\mathrm{C}_{6} \mathrm{H}_{5}$ & 4. & $2-\mathrm{OCHC}_{6} \mathrm{H}_{5}$ \\
\hline 5. & 3- $\mathrm{OCHC}_{6} \mathrm{H}_{5}$ & 6. & 4- $\mathrm{OCHC}_{6} \mathrm{H}_{5}$ & 7. & 3- $\mathrm{OCH}, 4-\mathrm{OCH}_{3}-\mathrm{C}_{6} \mathrm{H}_{4}$ & 8. & 5-Br-2-OH- $\mathrm{C}_{6} \mathrm{H}_{4}$ \\
\hline 9. & 2-Br- $\mathrm{C}_{6} \mathrm{H}_{5}$ & 10. & $3-\mathrm{Br}-\mathrm{C}_{6} \mathrm{H}_{5}$ & 11. & $4-\mathrm{Br}-\mathrm{C}_{6} \mathrm{H}_{5}$ & 12. & 2-Cl-6-F- $\mathrm{C}_{6} \mathrm{H}_{4}$ \\
\hline 13. & $4-\mathrm{Cl}-3-\mathrm{NO}_{2}$ & 14. & 2-Cl- $\mathrm{C}_{6} \mathrm{H}_{5}$ & 15. & $3-\mathrm{Cl}-\mathrm{C}_{6} \mathrm{H}_{5}$ & 16. & $4-\mathrm{Cl}-\mathrm{C}_{6} \mathrm{H}_{5}$ \\
\hline 17. & $2-\left(4-\mathrm{Cl}^{-} \mathrm{SC}_{6} \mathrm{H}_{5}\right)$ & 18. & $3-\mathrm{CN}-\mathrm{C}_{6} \mathrm{H}_{5}$ & 19. & $4-\mathrm{CN}-\mathrm{C}_{6} \mathrm{H}_{5}$ & 20. & $2,3-\mathrm{Cl}-\mathrm{C}_{6} \mathrm{H}_{4}$ \\
\hline 21. & $2,4-\mathrm{Cl}-\mathrm{C}_{6} \mathrm{H}_{4}$ & 22. & 2,6-Cl- $\mathrm{C}_{6} \mathrm{H}_{4}$ & 23. & $3,5-\mathrm{Cl}-\mathrm{C}_{6} \mathrm{H}_{4}$ & 24. & $4-\left(\mathrm{C}_{2} \mathrm{H}_{5}\right) 2 \mathrm{~N}-\mathrm{C}_{6} \mathrm{H}_{5}$ \\
\hline 25. & $2,6-\mathrm{F}-\mathrm{C}_{6} \mathrm{H}_{4}$ & 26. & $3,4-\mathrm{F}-\mathrm{C}_{6} \mathrm{H}_{4}$ & 27. & $2,3-\mathrm{OH}-\mathrm{C}_{6} \mathrm{H}_{4}$ & 28. & $2,4-\mathrm{OH}-\mathrm{C}_{6} \mathrm{H}_{4}$ \\
\hline 29. & $2,5-\mathrm{OH}-\mathrm{C}_{6} \mathrm{H}_{4}$ & 30 . & $3,4-\mathrm{OH}-\mathrm{C}_{6} \mathrm{H}_{4}$ & 31. & $2,3-\mathrm{OCH}_{3}-\mathrm{C}_{6} \mathrm{H}_{4}$ & 32. & $2,4-\mathrm{OCH}_{3}-\mathrm{C}_{6} \mathrm{H}_{4}$ \\
\hline 33. & $2,5-\mathrm{OCH}_{3}$ & 34. & $3,4-\mathrm{OCH}_{3}-\mathrm{C}_{6} \mathrm{H}_{4}$ & 35. & $3,5-\mathrm{OCH}_{3}-\mathrm{C}_{6} \mathrm{H}_{4}$ & 36. & $3,5-\mathrm{OCH}_{3}, 4-\mathrm{OH}-\mathrm{C}_{6} \mathrm{H}_{4}$ \\
\hline 37. & $2,4-\mathrm{CH}_{3}-\mathrm{C}_{6} \mathrm{H}_{4}$ & 38. & $3,5-\mathrm{CH}_{3}-\mathrm{C}_{6}$ & 39. & $3-\mathrm{OC}_{2} \mathrm{H}_{5}-4-\mathrm{OH}-\mathrm{C}_{6} \mathrm{H}_{4}$ & 40. & 2-F- $\mathrm{C}_{6} \mathrm{H}_{5}$ \\
\hline 41. & $3-\mathrm{F}-\mathrm{C}_{6} \mathrm{H}_{5}$ & 42. & $4-\mathrm{F}-\mathrm{C}_{6} \mathrm{H}_{5}$ & 43. & $2-\mathrm{OH}-\mathrm{C}_{6} \mathrm{H}_{5}$ & 44. & $3-\mathrm{OH}-\mathrm{C}_{6} \mathrm{H}_{5}$ \\
\hline 45. & $4-\mathrm{OH}-\mathrm{C}_{6} \mathrm{H}_{5}$ & 46. & $2-\mathrm{OH}, 3-\mathrm{OCH}_{3}-\mathrm{C}_{6} \mathrm{H}_{4}$ & 47. & $2-\mathrm{OH}, 4-\mathrm{OCH}_{3}-\mathrm{C}_{6} \mathrm{H}_{4}$ & 48. & $2-\mathrm{OH}, 5-\mathrm{OCH}_{3}-\mathrm{C}_{6} \mathrm{H}_{4}$ \\
\hline 49. & $\begin{array}{l}3-\mathrm{OH}, 4-\mathrm{OCH}_{3} \\
\mathrm{C}_{6} \mathrm{H}_{4}\end{array}$ & 50. & $4-\mathrm{OH}, 3-\mathrm{OCH}_{3}-5 \mathrm{NO}_{2}$ & 51. & $2-\mathrm{OH}-5 \mathrm{NO}_{2}$ & 52. & $4-\mathrm{CH}\left(\mathrm{CH}_{3}\right)_{2}-\mathrm{C}_{6} \mathrm{H}_{5}$ \\
\hline 53. & $2-\mathrm{OCH}_{3}-\mathrm{C}_{6} \mathrm{H}_{5}$ & 54. & $3-\mathrm{OCH}_{3}-\mathrm{C}_{6} \mathrm{H}_{5}$ & 55. & $4-\mathrm{OCH}_{3}-\mathrm{C}_{6} \mathrm{H}_{5}$ & 56. & $4-\mathrm{CH}_{3}-\mathrm{C}_{6} \mathrm{H}_{5}$ \\
\hline 57. & $2-\mathrm{CH}_{3}-\mathrm{C}_{6} \mathrm{H}_{5}$ & 58. & $3-\mathrm{CH}_{3}-\mathrm{C}_{6} \mathrm{H}_{5}$ & 59. & $4-\mathrm{SCH}_{3}-\mathrm{C}_{6} \mathrm{H}_{5}$ & 60. & $2-\mathrm{NO}_{2}-\mathrm{C}_{6} \mathrm{H}_{5}$ \\
\hline
\end{tabular}




\begin{tabular}{|c|l|c|l|r|l|l|l|}
\hline $\mathbf{6 1 .}$ & $3-\mathrm{NO}_{2}-\mathrm{C}_{6} \mathrm{H}_{5}$ & $\mathbf{6 2 .}$ & $4-\mathrm{NO}_{2}-\mathrm{C}_{6} \mathrm{H}_{5}$ & $\mathbf{6 3 .}$ & $3-\mathrm{OC}_{6} \mathrm{H}_{5}$ & $\mathbf{6 4 .}$ & $4-\mathrm{CH}\left(\mathrm{CH}_{3}\right)_{3}-\mathrm{C}_{6} \mathrm{H}_{5}$ \\
\hline $\mathbf{6 5 .}$ & $2,3,5-\mathrm{Cl}_{6} \mathrm{H}_{3}$ & $\mathbf{6 6 .}$ & $3,4,5-\mathrm{F}-\mathrm{C}_{6} \mathrm{H}_{3}$ & $\mathbf{6 7 .}$ & $4-\mathrm{CF}_{3} \mathrm{OCH}_{3}-\mathrm{C}_{6} \mathrm{H}_{4}$ & $\mathbf{6 8}$. & $4-\mathrm{CF}_{3}-\mathrm{C}_{6} \mathrm{H}_{5}$ \\
\hline $\mathbf{6 9 .}$ & $2,3,4-\mathrm{OCH}_{3}-$ & $\mathbf{7 0 .}$ & $3,4,5-\mathrm{OCH}_{3}-\mathrm{C}_{6} \mathrm{H}_{3}$ & $\mathbf{7 1 .}$ & $2,4,6-\mathrm{OCH}_{3}-\mathrm{C}_{6} \mathrm{H}_{3}$ & $\mathbf{7 2 .}$ & $3-\mathrm{NO}_{2}-\mathrm{C}_{6} \mathrm{H}_{5}$ \\
& $\mathrm{C}_{6} \mathrm{H}_{3}$ & & & & & \\
\hline $\mathbf{7 3 .}$ & $\mathrm{C}_{5} \mathrm{H}_{4} \mathrm{O}_{2}$ & $\mathbf{7 4 .}$ & $\mathrm{C}_{9} \mathrm{H}_{10} \mathrm{O}_{3}$ & $\mathbf{7 5 .}$ & $\mathrm{C}_{5} \mathrm{H}_{4} \mathrm{OS}$ & & \\
\hline
\end{tabular}




\subsection{Synthesis of selected ligands ${ }^{22}$}

\subsubsection{Synthesis of optimized thiazole derivatives}

\section{Step 1}

\section{Synthesis of 2-amino-5-aryl-5H thiazolo[ 4,3-b]-l,3,4-thiadiazole (1)}

In $25 \mathrm{ml}$ RBF Aldehyde (0.02M) and thioglycolic acid (0.02M), thiosemicarbazide $(0.022 \mathrm{M})$ and $10 \mathrm{ml}$ of concentrated $\mathrm{H}_{2} \mathrm{SO}_{4}$ were taken. The reaction mixture was mixed and left overnight and the resulting suspension was neutralized with $40 \% \mathrm{NaOH}$ solution till product gets precipitated out and this resulting compound 1was recrystallized from aqueous dioxane solution.

Synthesis of 1-phenyl-N-\{5-phenyl-5H-[1,3]thiazolo[4,3-b][1,3,4]thiadiazol-2-yl\}methanimine derivatives(2):

A solution of compound $1(0.01 \mathrm{M})$ in ethanol $(50 \mathrm{ml})$ was taken in RBF and stirred vigorously for 15 minutes to this resulting solution concentrated $\mathrm{H}_{2} \mathrm{SO}_{4}(2 \mathrm{ml})$ and aldehyde $(0.01 \mathrm{M})$ was added. The reaction mixture was irradiated in the synthetic microwave (Cata 4R) for 10min. The separated solid was filtered and recrystallized from ethanol.

Synthesis of 2-phenyl-3-\{5-phenyl-5H-[1,3]thiazolo[4,3-b][1,3,4]thiadiazol-2-yl\}-1,3thiazolidin-4-one derivatives (3):

In $25 \mathrm{ml}$ RBF compound, $2(0.01 \mathrm{M})$ and thioglycolic acid $(0.01 \mathrm{M})$ were taken and DMF $30 \mathrm{ml}$ was added and stirred. Resulting reaction mixture was irradiated in the microwave for $10 \mathrm{~min}$ at 750 watts. The reaction mixture was cooled to room temperature and resulting solid was separated and recrystallized from benzene to get compound 1 to 23 . Table 2 shown list of substituents in the synthesized set of molecules.

Table No. 2: List of Substituents in Synthesized set of molecules

\begin{tabular}{|c|l|l|c|}
\hline Sr. No. & \multicolumn{1}{|c|}{ R1 } & \multicolumn{1}{|c|}{ R2 } & Code \\
\hline 1. & $4-\mathrm{Br}_{-}-\mathrm{C}_{6} \mathrm{H}_{4}$ & $-\mathrm{C}_{6} \mathrm{H}_{4}$ & B3 \\
\hline 2. & $-\mathrm{C}_{6} \mathrm{H}_{4}$ & $4-\mathrm{Cl}_{-}-\mathrm{C}_{6} \mathrm{H}_{4}$ & $\mathrm{C} 13$ \\
\hline 3. & $-\mathrm{C}_{6} \mathrm{H}_{4}$ & $2-\mathrm{Cl}_{2}-\mathrm{C}_{6} \mathrm{H}_{4}$ & $\mathrm{C} 1$ \\
\hline 4. & $3,4-\left(\mathrm{OCH}_{3}\right)-\mathrm{C}_{6} \mathrm{H}_{4}$ & $2-\mathrm{Cl}_{-} \mathrm{C}_{6} \mathrm{H}_{4}$ & F1 \\
\hline 5. & $2-(\mathrm{OH})-\mathrm{C}_{6} \mathrm{H}_{4}$ & $3-\mathrm{Cl}_{4}-\mathrm{C}_{6} \mathrm{H}_{4}$ & O17 \\
\hline 6. & $-\mathrm{C}_{4} \mathrm{H}_{3} \mathrm{O}$ & $3-\mathrm{OCH}_{3}-4-\mathrm{OH}-\mathrm{C}_{6} \mathrm{H}_{3}$ & Q21 \\
\hline 7. & $4-\mathrm{N}\left(\mathrm{CH}_{3}\right)_{2}-\mathrm{C}_{6} \mathrm{H}_{4}$ & $4-\mathrm{Cl}_{4}-\mathrm{C}_{6} \mathrm{H}_{4}$ & L13 \\
\hline
\end{tabular}




\begin{tabular}{|c|c|c|c|}
\hline 8. & $2-(\mathrm{OH})-\mathrm{C}_{6} \mathrm{H}_{4}$ & $4-\mathrm{Cl}-\mathrm{C}_{6} \mathrm{H}_{4}$ & O13 \\
\hline 9. & $-\mathrm{C}_{4} \mathrm{H}_{3} \mathrm{O}$ & $2-(\mathrm{OH})-\mathrm{C}_{6} \mathrm{H}_{4}$ & Q24 \\
\hline 10. & $4-\mathrm{Cl}-\mathrm{C}_{6} \mathrm{H}_{4}$ & $4-(\mathrm{OH})-\mathrm{C}_{6} \mathrm{H}_{4}$ & M9 \\
\hline 11. & $4-\mathrm{F}-\mathrm{C}_{6} \mathrm{H}_{4}$ & $-\mathrm{C}_{4} \mathrm{H}_{3} \mathrm{O}$ & $\mathrm{H} 25$ \\
\hline 12. & $3-\mathrm{Cl}-\mathrm{C}_{6} \mathrm{H}_{4}$ & $\mathrm{~S}-\mathrm{C}_{4} \mathrm{H}_{3}$ & R26 \\
\hline 13. & $3-\left(\mathrm{OCH}_{3}\right)-4-(\mathrm{OH})-\mathrm{C}_{6} \mathrm{H}_{3}$ & $-\mathrm{C}_{6} \mathrm{H}_{4}$ & \\
\hline 14. & $3,5-\left(\mathrm{OCH}_{3}\right)_{2}-4-(\mathrm{OH}) \mathrm{C}_{6} \mathrm{H}_{2}$ & $-\mathrm{C}_{6} \mathrm{H}_{4}$ & U8 \\
\hline 15. & $3-\mathrm{Cl}-\mathrm{C}_{6} \mathrm{H}_{4}$ & $3-\mathrm{NO}_{2}-\mathrm{C}_{6} \mathrm{H}_{4}$ & A11 \\
\hline 16. & $2-\mathrm{Cl}-\mathrm{C}_{6} \mathrm{H}_{4}$ & $4-\mathrm{NO}_{2}-\mathrm{C}_{6} \mathrm{H}_{4}$ & A10 \\
\hline 17. & $-\mathrm{C}_{4} \mathrm{H}_{3} \mathrm{O}$ & $3-\mathrm{OCH}_{3}-\mathrm{C}_{6} \mathrm{H}_{4}$ & Q22 \\
\hline 18. & $-\mathrm{C}_{4} \mathrm{H}_{3} \mathrm{O}$ & $-\mathrm{C}_{7} \mathrm{H}_{5} \mathrm{O}_{2}$ & Q30 \\
\hline 19. & $3-\mathrm{Cl}-\mathrm{C}_{6} \mathrm{H}_{4}$ & $\mathrm{~S}-\mathrm{C}_{4} \mathrm{H}_{3}$ & R25 \\
\hline 20. & $4-\mathrm{Cl}-\mathrm{C}_{6} \mathrm{H}_{4}$ & $\mathrm{~S}-\mathrm{C}_{4} \mathrm{H}_{3}$ & M26 \\
\hline 21. & $3-\mathrm{Cl}-\mathrm{C}_{6} \mathrm{H}_{4}$ & $3,4-\left(\mathrm{OCH}_{3}\right)-\mathrm{C}_{6} \mathrm{H}_{4}$ & R6 \\
\hline 22. & $3-\left(\mathrm{OCH}_{3}\right)-\mathrm{C}_{6} \mathrm{H}_{4}$ & $4-\mathrm{Cl}-\mathrm{C}_{6} \mathrm{H}_{4}$ & W13 \\
\hline 23. & $3-\left(\mathrm{OCH}_{3}\right)-\mathrm{C}_{6} \mathrm{H}_{4}$ & $4-\mathrm{F}-\mathrm{C}_{6} \mathrm{H}_{4}$ & W8 \\
\hline
\end{tabular}

B3:3-[5-(4-bromophenyl)-5H-[1,3] thiazolo[4,3-b][1,3,4]thiadiazol-2-yl]-2-phenyl-1,3 thiazolidin-4-one

Color: Brown, Yield: $82 \%$, m. p.: $192-194^{\circ} \mathrm{C}$, MASS: $[\mathrm{M}+1]+474.94$, IR: $1752 \mathrm{~cm}^{-1}(\mathrm{C}=\mathrm{O}$ Str.), $1452 \mathrm{~cm}^{-1}(\mathrm{C}=\mathrm{C}), 2742.84 \mathrm{~cm}^{-1}$ (Ar. CH), $1580 \mathrm{~cm}^{-1}(-\mathrm{N}=\mathrm{CH})$ NMR: ${ }^{1} \mathrm{H}$ NMR (DMSO$d_{6}, 300 \mathrm{MHz}$,): $\delta=6.95-7.35$ (m, 9H, aromatic H), 4.95-5.90 (s, 2H, methine), 3.35 (s, 2H, methylene), 4.85 (s, $1 \mathrm{H}$, ethylene)

C13:2-(4-chlorophenyl)-3-\{5-phenyl-5H-[1,3]thiazolo[4,3-b][1,3,4]thiadiazol-2-yl\}-1,3thiazolidin-4-one

Color: Yellow, Yield: $80 \%$, m. p.: $178-180^{\circ} \mathrm{C}$, MASS: $[\mathrm{M}+1]+430.99$, IR: $1690 \mathrm{~cm}^{-1}(\mathrm{C}=\mathrm{O}$ Str.), $1435 \mathrm{~cm}^{-1}$ (C=C), $2842 \mathrm{~cm}^{-1}$ (Ar. CH), $1470 \mathrm{~cm}^{-1}$ (-N=CH), NMR: ${ }^{1} \mathrm{H}$ NMR (DMSO-d6, $300 \mathrm{MHz}$ ): $\delta=6.90-7.25$ (m, 9H, aromatic), 4.95-5.90 (s, 2H, methine), 3.38 (s, 2H, methylene), $4.95(\mathrm{~s}, 1 \mathrm{H}$, ethylene)

C1:2-(2-chlorophenyl)-3-\{5-phenyl-5H-[1,3]thiazolo[4,3-b][1,3,4]thiadiazol-2-yl\}-1,3thiazolidin-4-one 
Color: Brown, Yield: $75 \%$, m. p.: $186-188^{\circ} \mathrm{C}$, MASS: $[\mathrm{M}+1]+430.99$, IR: $1625 \mathrm{~cm}^{-1}(\mathrm{C}=\mathrm{O}$ Str.), $1400 \mathrm{~cm}^{-1}(\mathrm{C}=\mathrm{C}), 2830 \mathrm{~cm}^{-1}$ (Ar. CH), $1476 \mathrm{~cm}^{-1}(-\mathrm{N}=\mathrm{CH})$ NMR: ${ }^{1} \mathrm{H}$ NMR (DMSO-d 6,300 MHz,): $\delta=6.95-7.20$ (m, 9H, aromatic), 4.90-5.92 (s, 2H, methine), 3.30 (s, 2H, methylene), 4.90 (s, $1 \mathrm{H}$, ethylene)

F1: 2-(2-chlorophenyl)-3-[5-(3,4-dimethoxyphenyl)-5H-[1,3]thiazolo[4,3-b][1,3,4]thiadiazol-2yl]-1,3-thiazolidin-4-one

Color: Lemon, Yield: $89 \%$, m. p.: $162-164^{\circ} \mathrm{C}$, MASS: $[\mathrm{M}+1]+492.02$, IR: $1640 \mathrm{~cm}^{-1}(\mathrm{C}=\mathrm{O}$ Str.), $1490 \mathrm{~cm}^{-1}(\mathrm{C}=\mathrm{C}), 2790 \mathrm{~cm}^{-1}$ (Ar. CH), $1520 \mathrm{~cm}^{-1}(-\mathrm{N}=\mathrm{CH})$ NMR: ${ }^{1} \mathrm{H}$ NMR (DMSO-d, 300 $\mathrm{MHz}$ ): $\delta=6.52-7.15$ (m, 7H, aromatic), 4.95-5.90 (s, 2H, methine), 3.33 (s, 2H, methylene), 4.85 (s, 1H, ethylene), 3.75 (d, 6H, methyl)

O17: 2-(3-chlorophenyl)-3-[5-(2-hydroxyphenyl)-5H-[1,3]thiazolo[4,3-b][1,3,4]thiadiazol-2yl]-1,3-thiazolidin-4-one

Color: Yellow, Yield: $80 \%$, m. p.: $228-230^{\circ} \mathrm{C}$, MASS: [M+1]+447.97, IR: $1680 \mathrm{~cm}^{-1}(\mathrm{C}=\mathrm{O}$ Str.), $1500 \mathrm{~cm}^{-1}(\mathrm{C}=\mathrm{C}), 2810 \mathrm{~cm}^{-1}$ (Ar. $\left.\mathrm{CH}\right), 1580 \mathrm{~cm}^{-1}(-\mathrm{N}=\mathrm{CH})$ NMR: ${ }^{1} \mathrm{H}$ NMR (DMSO-d $d_{6}, 300$ $\mathrm{MHz},): \delta=6.62-7.20(\mathrm{~m}, 8 \mathrm{H}$, aromatic), $4.95(\mathrm{~s}, 1 \mathrm{H}$, methine), $5.92(\mathrm{~s}, 1 \mathrm{H}$, methine), $3.38(\mathrm{~s}, 1 \mathrm{H}$, methylene), 4.75 (s, 1H, ethylene), $6.05(\mathrm{~s}, 1 \mathrm{H}$, aromatic $\mathrm{C}-\mathrm{OH})$

Q21: 2-(3-ethoxy-4-hydroxyphenyl)-3-[5-(furan-2-yl)-5H-[1,3]thiazolo[4,3-b][1,3,4]thiadiazol2-yl]-1,3-thiazolidin-4-one

Color: Black, Yield: 78\%, m. p.:192-194 ${ }^{\circ}$, MASS: $[\mathrm{M}+1]+447.54$, IR: $1750 \mathrm{~cm}^{-1}(\mathrm{C}=\mathrm{O}$ Str.), $1540 \mathrm{~cm}^{-1}(\mathrm{C}=\mathrm{C}), 2840 \mathrm{~cm}^{-1}$ (Ar. $\left.\mathrm{CH}\right), 1600 \mathrm{~cm}^{-1}(-\mathrm{N}=\mathrm{CH})$ NMR: ${ }^{1} \mathrm{H}$ NMR (DMSO-d, 300 $\mathrm{MHz},): \delta=6.05-7.30(\mathrm{~m}, 6 \mathrm{H}$, aromatic benzene and furan), $4.75(\mathrm{~s}, 1 \mathrm{H}$, ethylene), 5.15-5.92 (s, $2 \mathrm{H}$, methine), $3.30(\mathrm{~s}, 2 \mathrm{H}$, methylene), 3.95 (s, 2H, methylene), 1.58 (s, 3H, methyl), $5.02(\mathrm{~s}, 1 \mathrm{H}$ aromatic $\mathrm{C}-\mathrm{OH})$

L13:2-(4-chlorophenyl)-3-\{5-[4-(dimethylamino)phenyl]-5H-[1,3]thiazolo[4,3b][1,3,4]thiadiazol-2-yl\}-1,3-thiazolidin-4-one

Color: Brown, Yield: $67 \%$, m. p.: 156-158 ${ }^{\circ}$ C, MASS: [M+1]+475.04, IR: $1652 \mathrm{~cm}^{-1}$ (C=O Str.), $1538 \mathrm{~cm}^{-1}(\mathrm{C}=\mathrm{C}), 2800 \mathrm{~cm}^{-1}$ (Ar. CH), $1530 \mathrm{~cm}^{-1}(-\mathrm{N}=\mathrm{CH})$ NMR: ${ }^{1} \mathrm{H}$ NMR (DMSO-d 6,300 MHz,): $\delta=6.45-7.20(\mathrm{~m}, 8 \mathrm{H}$, aromatic benzene), $4.80(\mathrm{~s}, 1 \mathrm{H}$, ethylene), 4.95-5.80 (s, 2H, methine), 3.30 (s, 2H, methylene), $2.95\left(\mathrm{~s}, 6 \mathrm{H}\right.$, methyl $\left.\mathrm{N}-\mathrm{CH}_{3}\right)$

O13: 2-(4-chlorophenyl)-3-[5-(2-hydroxyphenyl)-5H-[1,3]thiazolo[4,3-b][1,3,4]thiadiazol-2yl]-1,3-thiazolidin-4-one 
Color: Brown, Yield: 67\%, m. p.: 236-238 ${ }^{\circ} \mathrm{C}$, MASS: [M+1]+447.97, IR: $1760 \mathrm{~cm}^{-1}$ (C=O Str.), $1540 \mathrm{~cm}^{-1}(\mathrm{C}=\mathrm{C}), 2830 \mathrm{~cm}^{-1}$ (Ar. $\left.\mathrm{CH}\right), 1680 \mathrm{~cm}^{-1}(-\mathrm{N}=\mathrm{CH})$ NMR: ${ }^{1} \mathrm{H}$ NMR (DMSO- $d_{6}, 300$ MHz,): $\delta=6.65-7.25(\mathrm{~m}, 8 \mathrm{H}$, aromatic benzene), 4.85 (s, $1 \mathrm{H}$, ethylene), 4.05-4.70 (s, 2H, methine), 3.28 (s, 2H, methylene), 5.25 (s, 1H, aromatic C-OH)

Q24: 3-[5-(furan-2-yl)-5H-[1,3]thiazolo[4,3-b][1,3,4]thiadiazol-2-yl]-2-(2-hydroxyphenyl)-1,3thiazolidin-4-one

Color: Black, Yield: $75 \%$, m. p.: 216-218 ${ }^{\circ}$ C, MASS: [M+1]+403.49, IR: $1740 \mathrm{~cm}^{-1}$ (C=O Str.), $1623 \mathrm{~cm}^{-1}(\mathrm{C}=\mathrm{C}), 2810 \mathrm{~cm}^{-1}$ (Ar. CH), $1680 \mathrm{~cm}^{-1}(-\mathrm{N}=\mathrm{CH})$, NMR: ${ }^{1} \mathrm{H}$ NMR (DMSO-d 6,300 $\mathrm{MHz},) \delta=6.60-7.30(\mathrm{~m}, 7 \mathrm{H}$, aromatic benzene and furan), 4.80 (s, $1 \mathrm{H}$, ethylene), 5.20-5.80 (s, $2 \mathrm{H}$, methine), 3.30 (s, $2 \mathrm{H}$, methylene), $5.25(\mathrm{~s}, 1 \mathrm{H}$, aromatic $\mathrm{C}-\mathrm{OH})$

M9: 3-[5-(4-chlorophenyl)-5H-[1,3]thiazolo[4,3-b][1,3,4]thiadiazol-2-yl]-2-(4-hydroxyphenyl)-

\section{1,3-thiazolidin-4-one}

Color: Brown, Yield: $88 \%$, m. p.: $138-140^{\circ} \mathrm{C}$, MASS: $[\mathrm{M}+1]+447.97$, IR: $1700 \mathrm{~cm}^{-1}$ (C $=\mathrm{O}$ Str.), $1640 \mathrm{~cm}^{-1}(\mathrm{C}=\mathrm{C}), 2790 \mathrm{~cm}^{-1}$ (Ar. CH), $1650 \mathrm{~cm}^{-1}(-\mathrm{N}=\mathrm{CH})$, NMR: ${ }^{1} \mathrm{H}$ NMR (DMSO-d 6,300 $\mathrm{MHz},): \delta=6.55-7.25(\mathrm{~m}, 8 \mathrm{H}$, aromatic benzene), $4.85(\mathrm{~s}, 1 \mathrm{H}$, ethylene), 5.10-5.85 (s, 2H, methine), 3.38 (s, 2H, methylene), 6.05 (s, $1 \mathrm{H}$, aromatic $\mathrm{C}-\mathrm{OH})$

H25: 3-[5-(4-fluorophenyl)-5H-[1,3]thiazolo[4,3-b][1,3,4]thiadiazol-2-yl]-2-(furan-2-yl)-1,3thiazolidin-4-one

Color: Yellow , Yield: $70 \%$, m. p.: $122-124^{\circ} \mathrm{C}$, MASS: $[\mathrm{M}+1]+405.48$, IR: $1688 \mathrm{~cm}^{-1}(\mathrm{C}=\mathrm{O}$ Str.), $1589 \mathrm{~cm}^{-1}(\mathrm{C}=\mathrm{C}), 2690 \mathrm{~cm}^{-1}$ (Ar. CH), $1580 \mathrm{~cm}^{-1}(-\mathrm{N}=\mathrm{CH})$ NMR: ${ }^{1} \mathrm{H}$ NMR (DMSO-d 6,300 $\mathrm{MHz}$ ): $\delta=6.30-7.05(\mathrm{~m}, 7 \mathrm{H}$, aromatic benzene and furan), 4.82 (s, $1 \mathrm{H}$, ethylene), 5.10-5.85 (s, $2 \mathrm{H}$, methine), $3.35(\mathrm{~s}, 2 \mathrm{H}$, methylene)

R26: 3-[5-(3-chlorophenyl)-5H-[1,3]thiazolo[4,3-b][1,3,4]thiadiazol-2-yl]-2-(thiophen-2-yl)1,3-thiazolidin-4-one

Color: Yellow, Yield: $72 \%$, m. p.: $206-208^{\circ} \mathrm{C}$, MASS: $[\mathrm{M}+1]+437.99$, IR: $1750 \mathrm{~cm}^{-1}(\mathrm{C}=\mathrm{O}$ Str.), $1670 \mathrm{~cm}^{-1}(\mathrm{C}=\mathrm{C}), 2560 \mathrm{~cm}^{-1}$ (Ar. CH), $1540 \mathrm{~cm}^{-1}(-\mathrm{N}=\mathrm{CH})$ NMR: ${ }^{1} \mathrm{H}$ NMR (DMSO-d $d_{6}, 300$ $\mathrm{MHz}$ ): $\delta=6.60-7.10$ (m, 7H, aromatic benzene and thiophene), 4.85 (s, 1H, ethylene), 4.95-5.90 (s, 2H, methine), $3.30(\mathrm{~s}, 2 \mathrm{H}$, methylene)

V3: 3-[5-(3-ethoxy-4-hydroxyphenyl)-5H-[1,3]thiazolo[4,3-b][1,3,4]thiadiazol-2-yl]-2-phenyl1,3-thiazolidin-4-one 
Color: Brown, Yield: $83 \%$, m. p.: $180-182^{\circ} \mathrm{C}$, MASS: [M+1]+457.58, IR: $1620 \mathrm{~cm}^{-1}$ (C=O Str.), $1560 \mathrm{~cm}^{-1}(\mathrm{C}=\mathrm{C}), 2860 \mathrm{~cm}^{-1}$ (Ar. CH), $1490 \mathrm{~cm}^{-1}(-\mathrm{N}=\mathrm{CH})$ NMR: ${ }^{1} \mathrm{H}$ NMR (DMSO-d 6,300 $\mathrm{MHz},) \delta=6.45-7.14(\mathrm{~m}, 8 \mathrm{H}$, aromatic benzene), $4.80(\mathrm{~s}, 1 \mathrm{H}$, ethylene), 4.90-5.85 (s, 2H, methine), 3.35 (s, 2H, methylene), 5.95 (s, 1H, aromatic C-OH), 3.98 (s, 2H, methylene), 1.75 (s, $3 \mathrm{H}$, methyl)

U8: 3-[5-(4-hydroxy-3,5-dimethoxyphenyl)-5H-[1,3]thiazolo[4,3-b][1,3,4]thiadiazol-2-yl]-2phenyl-1,3-thiazolidin-4-one

Color: Yellow, Yield: $80 \%$, m. p.: $230-232^{\circ} \mathrm{C}$, MASS: $[\mathrm{M}+1]+473.58$, IR: $1688 \mathrm{~cm}^{-1}(\mathrm{C}=\mathrm{O}$ Str.), $1562 \mathrm{~cm}^{-1}(\mathrm{C}=\mathrm{C}), 2883 \mathrm{~cm}^{-1}$ (Ar. CH), $1489 \mathrm{~cm}^{-1}(-\mathrm{N}=\mathrm{CH}) \quad$ NMR: ${ }^{1} \mathrm{H}$ NMR (DMSO- $d_{6}$, $300 \mathrm{MHz}$ ): $\delta=5.95-7.10$ (m, 7H, aromatic benzene), 4.85 (s, 1H, ethylene), 4.92-5.95 (s, 2H, methine), $3.28(\mathrm{~s}, 2 \mathrm{H}$, methylene), $5.98(\mathrm{~s}, 1 \mathrm{H}$, aromatic $\mathrm{C}-\mathrm{OH}), 3.85(\mathrm{~s}, 6 \mathrm{H}$, methoxy)

A11: 3-[5-(3-chlorophenyl)-5H-[1,3]thiazolo[4,3-b][1,3,4]thiadiazol-2-yl]-2-(3-nitrophenyl)-

\section{1,3-thiazolidin-4-one}

Color: Brown, Yield: 69 \%, m. p.: 146-148 ${ }^{\circ}$, MASS: $[\mathrm{M}+1]+476.97$, IR: $1679 \mathrm{~cm}^{-1}$ (C=O Str.), $1575 \mathrm{~cm}^{-1}(\mathrm{C}=\mathrm{C}), 2896 \mathrm{~cm}^{-1}$ (Ar. CH), $1523 \mathrm{~cm}^{-1}(-\mathrm{N}=\mathrm{CH})$. NMR: ${ }^{1} \mathrm{H}$ NMR (DMSO-d 6,300 $\mathrm{MHz},) \delta=6.90-7.95(\mathrm{~m}, 8 \mathrm{H}$, aromatic benzene), 4.75 (s, $1 \mathrm{H}$, ethylene), 4.90-5.92 (s, 2H, methine), 3.33 (s, 2H, methylene)

A10: 3-[5-(2-chlorophenyl)-5H-[1,3]thiazolo[4,3-b][1,3,4]thiadiazol-2-yl]-2-(4-nitrophenyl)1,3-thiazolidin-4-one

Color: Brown, Yield: 70\%, m.p.: 174-176 C, MASS: [M+1]+476.97, IR: $1590 \mathrm{~cm}^{-1}$ (C=O Str.), $1456 \mathrm{~cm}^{-1}(\mathrm{C}=\mathrm{C}), 2675 \mathrm{~cm}^{-1}$ (Ar. $\left.\mathrm{CH}\right), 1563 \mathrm{~cm}^{-1}(-\mathrm{N}=\mathrm{CH})$. NMR: ${ }^{1} \mathrm{H}$ NMR (DMSO-d, 300 $\mathrm{MHz},) \delta=7.04-8.05(\mathrm{~m}, 8 \mathrm{H}$, aromatic benzene), 4.85 (s, $1 \mathrm{H}$, ethylene), 4.95-5.90 (s, $2 \mathrm{H}$, methine), $3.28(\mathrm{~s}, 2 \mathrm{H}$, methylene)

Q22: 3-[5-(furan-2-yl)-5H-[1,3]thiazolo[4,3-b][1,3,4]thiadiazol-2-yl]-2-(3-methoxyphenyl)-1,3thiazolidin-4-one

Color: Black, Yield: 78 \%, m. p.: 176-178 C, MASS: $[\mathrm{M}+1]+417.52$, IR: $1639 \mathrm{~cm}^{-1}$ (C=O Str.), $1420 \mathrm{~cm}^{-1}(\mathrm{C}=\mathrm{C}), 2640 \mathrm{~cm}^{-1}$ (Ar. CH), $1570 \mathrm{~cm}^{-1}(-\mathrm{N}=\mathrm{CH})$. NMR: ${ }^{1} \mathrm{H}$ NMR (DMSO-d 6,300 $\mathrm{MHz}$ ): $\delta=6.50-7.30$ (m, 7H, aromatic benzene and furan), 4.80 (s, 1H, ethylene), 5.10-5.95 (s, $2 \mathrm{H}$, methine), 3.30 (s, 2H, methylene), 3.75 (s, 3H, methoxy)

Q30: 2-(2H-1,3-benzodioxol-5-yl)-3-[5-(furan-2-yl)-5H-[1,3]thiazolo[4,3-b][1,3,4]thiadiazol-2yl]-1,3-thiazolidin-4-one 
Color: Black, Yield: $70 \%$, m. p.: 240-242 ${ }^{\circ}$, MASS: $[\mathrm{M}+1]+431.50$, IR: $1782 \mathrm{~cm}^{-1}$ (C=O Str.), $1560 \mathrm{~cm}^{-1}(\mathrm{C}=\mathrm{C}), 2785 \mathrm{~cm}^{-1}$ (Ar. $\left.\mathrm{CH}\right), 1630 \mathrm{~cm}^{-1}(-\mathrm{N}=\mathrm{CH})$. NMR: ${ }^{1} \mathrm{H}$ NMR (DMSO-d, 300 $\mathrm{MHz},{ }: \delta=6.45-7.25(\mathrm{~m}, 6 \mathrm{H}$, aromatic benzene and furan), 4.82 (s, $1 \mathrm{H}$, ethylene), 5.15-5.85 (s, 2H, methine), 3.28 (s, 2H, methylene), 5.95 (s, 2H, 1,3-dioxole)

R25: 3-[5-(3-chlorophenyl)-5H-[1,3] thiazolo[4,3-b][1,3,4]thiadiazol-2-yl]-2-(thiophen-2-yl)1,3-thiazolidin-4-one

Color: Yellow, Yield: $82 \%$, m. p.: $234-236^{\circ} \mathrm{C}$, MASS: $[\mathrm{M}+1]+437.99$ IR: $1700 \mathrm{~cm}^{-1}(\mathrm{C}=\mathrm{O}$ Str.), $1590 \mathrm{~cm}^{-1}(\mathrm{C}=\mathrm{C}), 2693 \mathrm{~cm}^{-1}$ (Ar. CH), $1578 \mathrm{~cm}^{-1}(-\mathrm{N}=\mathrm{CH})$. NMR: ${ }^{1} \mathrm{H}$ NMR (DMSO-d 6 , $300 \mathrm{MHz}$,): $\delta=6.65-7.05$ (m, 7H, aromatic benzene and thiophene), 4.85 (s, 1H, ethylene), $4.95-$ 5.95 (s, 2H, methine), 3.32 (s, 2H, methylene)

M26: 3-[5-(4-chlorophenyl)-5H-[1,3]thiazolo[4,3-b][1,3,4]thiadiazol-2-yl]-2-(thiophen-2-yl)1,3-thiazolidin-4-one

Color: Brown, Yield: 70\%, m. p.: 210-212 ${ }^{\circ}$ C, MASS: [M+1]+437.99, IR: $1665 \mathrm{~cm}^{-1}$ (C=O Str.), $1545 \mathrm{~cm}^{-1}(\mathrm{C}=\mathrm{C}), 2530 \mathrm{~cm}^{-1}$ (Ar. $\left.\mathrm{CH}\right), 1540 \mathrm{~cm}^{-1}(-\mathrm{N}=\mathrm{CH})$. NMR: ${ }^{1} \mathrm{H}$ NMR (DMSO-d, 300 $\mathrm{MHz},): \delta=6.60-7.25$ (m, 7H, aromatic benzene and thiophene), 4.82 (s, 1H, ethylene), 4.90-5.92 (s, 2H, methine), 3.35 (s, 2H, methylene)

R6: 3-[5-(3-chlorophenyl)-5H-[1,3]thiazolo[4,3-b][1,3,4]thiadiazol-2-yl]-2-(3,4 dimethoxyphenyl)-1,3-thiazolidin-4-one

Color: Brown, Yield: $85 \%$, m. p.: 226-228 ${ }^{\circ} \mathrm{C}$, MASS: [M+1]+492.02, IR: $1675 \mathrm{~cm}^{-1}$ (C $=\mathrm{O}$ Str.), $1553 \mathrm{~cm}^{-1}(\mathrm{C}=\mathrm{C}), 2542 \mathrm{~cm}^{-1}$ (Ar. CH), $1580 \mathrm{~cm}^{-1}(-\mathrm{N}=\mathrm{CH})$. NMR: ${ }^{1} \mathrm{H}$ NMR (DMSO-d6, 300 $\mathrm{MHz}$ ): $\delta=6.50-7.15$ ( $\mathrm{m}, 7 \mathrm{H}$, aromatic benzene), 4.82 (s, 1H, ethylene), 4.92-5.85 (s, 2H, methine), 3.33 (s, 2H, methylene), 3.75 (s, $6 \mathrm{H}$, methoxy)

W13: 2-(4-chlorophenyl)-3-[5-(3-methoxyphenyl)-5H-[1,3]thiazolo[4,3-b][1,3,4]thiadiazol-2yl]-1,3-thiazolidin-4-one

Color: White, Yield: $78 \%$, m. p.: $170-172^{\circ} \mathrm{C}$, MASS: $[\mathrm{M}+1]+462.00$, IR: $1600 \mathrm{~cm}^{-1}$ (C=O Str.), $1532 \mathrm{~cm}^{-1}(\mathrm{C}=\mathrm{C}), 2520 \mathrm{~cm}^{-1}$ (Ar. $\left.\mathrm{CH}\right), 1560 \mathrm{~cm}^{-1}(-\mathrm{N}=\mathrm{CH}) . \mathbf{N M R}{ }^{1} \mathrm{H}$ NMR (DMSO-d, 300 $\mathrm{MHz}$,): $\delta=6.55-7.20$ ( $\mathrm{m}, 8 \mathrm{H}$, aromatic benzene), 4.85 (s, 1H, ethylene), 4.92-5.88 (s, 2H, methine), 3.30 (s, $2 \mathrm{H}$, methylene), 3.78 (s, $3 \mathrm{H}$, methoxy)

W8: 2-(4-fluorophenyl)-3-[5-(3-methoxyphenyl)-5H-[1,3]thiazolo[4,3-b][1,3,4]thiadiazol-2-yl]1,3-thiazolidin-4-one

Color: Brown, Yield: $80 \%$, m. p.: $160-162^{\circ} \mathrm{C}$, MASS: $[\mathrm{M}+1]+445.55$ IR: $1750 \mathrm{~cm}^{-1}$ (C $=\mathrm{O}$ Str.), 
$1682 \mathrm{~cm}^{-1}(\mathrm{C}=\mathrm{C}), 2540 \mathrm{~cm}^{-1}$ (Ar. $\left.\mathrm{CH}\right), 1670 \mathrm{~cm}^{-1}(-\mathrm{N}=\mathrm{CH})$. NMR: ${ }^{1} \mathrm{H}$ NMR (DMSO-d, 300

$\mathrm{MHz},): \delta=6.65-7.05(\mathrm{~m}, 8 \mathrm{H}$, aromatic benzene), $4.80(\mathrm{~s}, 1 \mathrm{H}$, ethylene), 4.85-5.90 (s, $2 \mathrm{H}$, methine), 3.34 (s, 2H, methylene), 3.65 (s, 3H, methoxy)

\subsection{Antitubercular Activity:}

The synthesized molecules were evaluated for antimycobacterial activity by culturing Mycobacterium smegmatis (NCIM 5138) on Middlebrook $7 \mathrm{H} 9$ broth (Difco) containing $0.5 \%$ albumin, $0.085 \% \mathrm{NaCl}, 0.2 \%$ glucose, $0.05 \%$ Tween 80 , and $0.5 \%$ glycerol at $37^{\circ} \mathrm{C}$ for $48 \mathrm{hr}$ to mid-log phase (optical density at $600 \mathrm{~nm}\left[\mathrm{OD}_{600}\right]=0.5$ ) MICs of antibiotics against Mycobacterium smegmatis were determined in Middlebrook 7H9 broth by the standard microdilution method. All synthesized derivatives were dissolved in dimethyl sulfoxide and utilized for antimycobacterial assay in concentration range of $(3.25-1000 \mu \mathrm{gm} / \mathrm{ml})$

\section{RESULTS AND DISCUSSION}

\section{Pocket modeling of SEC of Mycobacterium tuberculosis:}

The binding pocket analysis was performed on the X-ray structure of the SEC from MTB downloaded from free protein database www.rcsb.org. Proviz module of Vlife MDS 4.4 was utilized to perform the pocket analysis. Electrostatic and hydrophobic map of the binding pocket of SEC was generated to identify the relative orientation of critical amino acids. Pocket modeling of SEC revealed the binding pocket of SEC is highly hydrophobic and ASP224, HIS534, and LYS115 are the three important amino acids required for the ATP binding. Fig. 2 showing the hydrophobic map of the active site of SEC of Mycobacterium tuberculosis. SEC runs protein export, which is driven by ATP as the energy source, inhibition of this ATP binding, or blocking three amino acids will inhibit the SEC and ultimately protein export. Binding pocket of SEC was found to be highly hydrophobic and U shaped. Molecules were designed with an intent so that they will retain necessary hydrophobicity and relative conformation with respect to the SEC of MTB. Molecules are developed keeping thiazole as the template, thiazole derivatives due presence of two heteroatoms which will act as an anchor and two aromatic rings will act as wings to the nucleus and capable of achieving the bioactive $\mathrm{U}$ or $\mathrm{V}$-shaped conformation which is complementary to the binding site of SEC. Aromatic benzaldehydes are utilized to manipulate the required hydrophobic characters and from aromatic interaction with Histidine 534.

Screening based on the drug-like properties: 
Pharmacokinetic properties of the molecules are an important factor in the conversion of any New Chemical Entity to the drug. Pre-assessment of this drug like properties is played a vital role in the selection of potential drug-like candidates from designed data set of the molecules. All the molecules in the designed set of thiazole derivatives were assessed for the Lipinski parameters like molecular weight, H-bond acceptor, H-bond donor, Rotatable bond logp and Predicted oral absorption. 23 derivatives were found to have desired pharmacokinetic properties and predicted oral absorption $<70 \%$. The selected 23 derivatives with the drug-like properties were given in the following table 3 .

Table No. 3: Table showing the molecules having the desired drug-like properties

\begin{tabular}{|c|c|c|c|c|c|c|c|c|}
\hline Sr. No. & $\begin{array}{c}\text { Compound } \\
\text { Code }\end{array}$ & Mole. Wt. & $\begin{array}{c}\text { H- } \\
\text { Acce }\end{array}$ & $\begin{array}{c}\text { H- } \\
\text { Donor } \\
\text { Count }\end{array}$ & RBC & logP & $\begin{array}{c}\text { TPSA } \\
(\AA)\end{array}$ & $\begin{array}{c}\text { Pre. \% } \\
\text { Oral } \\
\text { Abs. }\end{array}$ \\
\hline 1 & A10 & 476.988 & 4 & 0 & 4 & 4.738 & 44.81 & 93.54 \\
\hline 2 & A11 & 476.988 & 4 & 0 & 4 & 4.738 & 81.4 & 80.91 \\
\hline 3 & B3 & 476.442 & 1 & 0 & 3 & 4.939 & 35.57 & 96.72 \\
\hline 4 & C1 & 431.99 & 1 & 0 & 3 & 4.83 & 35.57 & 96.72 \\
\hline 5 & C13 & 431.99 & 1 & 0 & 3 & 4.83 & 35.57 & 96.72 \\
\hline 6 & F1 & 492.043 & 3 & 0 & 7 & 4.847 & 54.04 & 90.35 \\
\hline 7 & H25 & 405.498 & 2 & 0 & 3 & 3.908 & 46.57 & 85.26 \\
\hline 8 & L13 & 475.059 & 2 & 0 & 6 & 4.896 & 41.37 & 94.72 \\
\hline 9 & M9 & 447.99 & 2 & 1 & 4 & 4.535 & 58.63 & 88.77 \\
\hline 10 & M26 & 438.019 & 1 & 0 & 3 & 4.891 & 35.57 & 96.72 \\
\hline 11 & O13 & 447.99 & 2 & 1 & 4 & 4.535 & 58.63 & 88.77 \\
\hline 12 & O17 & 447.99 & 2 & 1 & 4 & 4.535 & 58.63 & 88.77 \\
\hline 13 & Q21 & 447.56 & 3 & 1 & 7 & 3.873 & 81 & 81.05 \\
\hline 14 & Q22 & 417.534 & 2 & 0 & 5 & 3.778 & 57.95 & 89.00 \\
\hline 15 & Q24 & 433.533 & 3 & 1 & 6 & 3.483 & 81 & 81.05 \\
\hline 16 & Q30 & 431.517 & 1 & 0 & 3 & 3.498 & 67.18 & 85.82 \\
\hline 17 & R6 & 492.043 & 3 & 0 & 7 & 4.847 & 54.04 & 90.35 \\
\hline
\end{tabular}




\begin{tabular}{|c|c|c|c|c|c|c|c|c|}
\hline 18 & R25 & 421.952 & 1 & 0 & 3 & 4.423 & 48.71 & 92.19505 \\
\hline 19 & R26 & 438.019 & 1 & 0 & 3 & 4.891 & 35.57 & 96.72 \\
\hline 20 & U8 & 491.588 & 5 & 1 & 8 & 4.038 & 77.1 & 82.40 \\
\hline 21 & V3 & 457.598 & 3 & 1 & 7 & 4.28 & 67.86 & 85.58 \\
\hline 22 & W8 & 445.562 & 3 & 0 & 5 & 4.324 & 44.81 & 93.54 \\
\hline 23 & W13 & 462.017 & 2 & 0 & 5 & 4.838 & 44.81 & 93.54 \\
\hline
\end{tabular}

\section{Molecular Docking:}

Molecular docking was utilized to predict the potential active inhibitors from the designed set of ligands. Grip-based docking analysis was performed with maintaining the SEC in rigid conformation while ligands in flexible conformation. All the design set of molecules were found to be binding to the same binding site to that of ATP in SEC. The designed set of molecules were found to be interacting with HIS 534 and ASP224 via formation with hydrogen bond interaction and pi-stacking interaction. Binding interactions of selected 23 molecules were summarized in the following table no. 4, whereas Fig. 3, 4, 5 and 6 showing most active conformation of molecule M26, U8, R26 and A10 (Ball and Stick) with PDB ID 4UAQ respectively.

Table No. 4: Binding interactions and energy of docked molecules

\begin{tabular}{|c|c|c|c|c|}
\hline Sr. No. & Compound Code & Binding Energy & H-bond & Pi-Stacking \\
\hline 1. & A10 & -33.43 & HIS534 & HIS534 \\
\hline 2. & A11 & -0.12 & HIS534 & HIS534 \\
\hline 3. & B3 & -35.40 & HIS534 & HIS534 \\
\hline 4. & C1 & -7.41 & ASP224 & HIS534 \\
\hline 5. & C13 & -2.31 & HIS534 & HIS534 \\
\hline 6. & F1 & -33.32 & HIS534 & \\
\hline 7. & L13 & -8.74 & HIS534 & HIS534 \\
\hline 8. & M9 & -37.54 & ASP219 & HIS534 \\
\hline 9. & M26 & -38.54 & ASP224 & HIS534 \\
\hline 10. & O13 & -11.91 & GLN498 & HIS534 \\
\hline
\end{tabular}




\begin{tabular}{|c|c|c|c|c|}
\hline 11. & O17 & -9.81 & HIS534 & HIS534 \\
\hline 12. & Q21 & 23.92 & ASP219 & HIS534 \\
\hline 13. & Q22 & -11.97 & HIS534 & HIS534 \\
\hline 14. & Q24 & -11.03 & HIS534 & HIS534 \\
\hline 15. & Q30 & -3.89 & HIS534 & HIS534 \\
\hline 16. & R25 & -2.51 & HIS534 & HIS534 \\
\hline 17. & R26 & -4.66 & ASP224 & HIS534 \\
\hline 18. & R6 & -11.74 & HIS534 & HIS534 \\
\hline 19. & U8 & -0.21 & HIS534 & HIS534 \\
\hline 20. & V3 & -36.40 & HIS534 & HIS534 \\
\hline 21. & H25 & -8.37 & HIS534 & HIS534 \\
\hline 22. & W8 & -33.40 & ASP224 & HIS534 \\
\hline 23. & W13 & -31.56 & HIS534 & HIS534 \\
\hline
\end{tabular}

\section{Biological activity:}

The anti-mycobacterial activity of the synthesized derivatives was determined against the standard Mycobacterium smegmatis (NCIM No: 5138). The anti-mycobacterial activity of the synthesized derivatives was determined against the standard Mycobacterium smegmatis (NCIM No: 5138). Mycobacterium smegmatis is an organism belonging to Mycobacterium family having nearly about $80 \%$ genome similarity with Mycobacterium tuberculosis. Isoniazid is utilized as the positive standard for the anti-mycobacterial activity. Minimum inhibitory concentration (MIC) of the all23 derivatives are summarized in Fig 7. All the compounds have shown good to moderate activity against the tested strain. Compound R 26, Q30 and M9 are showed maximum antitubercular activity (MIC: $62.5 \mu \mathrm{gm} / \mathrm{ml}$ ). Excellent activity of the M9 and R 26 indicates halogens are having the positive effect on the antitubercular activity, which activity of Q30 is interesting finding which indicates substitution of the heterocyclic nucleus will also potentiate the activity. Derivative L13 showed good activity (MIC: $125 \mu \mathrm{gm} / \mathrm{ml}$ ), which justifies the substitution of halogen in the aromatic ring. A10, C13, F1, O13, O17, Q21, Q22, Q24, R25, R6, U8, and W13 are moderately active compounds with (MIC: $500 \mu \mathrm{gm} / \mathrm{ml}$ ).

\section{Conclusion:}


Attempts to design and develop molecules targeting secretory systems of Mycobacterium tuberculosis yielded following significant findings. Identification and validation of secretory systems as targets for anti-tubercular drug design and discovery has been successfully carried out. Out of three protein export systems associated with Mycobacterium tuberculosis SEC protein export system has been utilized successfully for the development of antitubercular against which are selective and can be utilized against all resistant form of tuberculosis. Protein export systems are conserved systems and they are not having any isoform in human so targeting protein export systems will be the potential route for development of selective and active antitubercular agents. Pocket modeling of the active site or binding site of SEC revealed the interesting fact about size and shape of the binding pocket of SEC and which signified the development of hydrophobic ligands for binding with SEC of Mycobacterium tuberculosis. Based on pocket modeling data and Literature survey total 5184 molecules were designed around thiazole scaffold via the change in the different aromatic benzaldehyde structures. 5625 designed molecules were further scrutinized via Lipinski drug-like properties and toxicity profile using OCHEM and for binding efficiency using molecular docking analysis. Results of above all three analysis yielded 23 potent, selective molecules which are successfully synthesized via reaction of an aromatic aldehyde, thiosemicarbazide and thioglycolic acid. All the molecules are successfully synthesized using microwave assisted synthesis which improved the yield and reduced to the time of reaction than the procedures reported in the literature. All the synthesized 23 derivatives were characterized via all physicochemical methods, Melting point and IR, and NMR spectroscopy. Halogen substituted derivatives showed significant activity which indicates substitution of an electron withdrawing group in aryl ring will potentiate anti-mycobacterial activity. M26, U8 and R26 molecules have significant desirable biological activity and specific interactions with SEC, Further optimization of these leads is necessary for the development of potential antitubercular drug like candidates. These potential drug candidates with specific SEC inhibitory properties have resulted from the utilization of integration of pocket modeling and virtual screening. 


\section{References:}

1. R. Abagyan, M. Totrov, High-throughput Docking for Lead Generation. Curr Opin in Chem Bio. 4(2001), 375-382.

2. S. Alam, F. Khan, QSAR, and Docking studies on Xanthone Derivatives for Anticancer Activity Targeting DNA topoisomerase II $\alpha$. Drug Des Devel and The. 8 (2014), 183-195.

3. L.E. Bermudez, J. Goodman, Mycobacterium tuberculosis invades and replicates within Type II alveolar cells. Infect Immun. 64(1996) 1400-1406.

4. M. S. Bhatia, K. B. Ingale, P. B. Choudhari, N. M. Bhatia, R. L. Sawant, Application quantum and physicochemical molecular descriptors utilizing principal components to study the mode of anticoagulant activity of pyridyl chromen-2-one derivatives. Bioorg. Med. Chem. 17(2009), 1654-1662.

5. S. Borrell, S. Gagneux. Infectiousness, reproductive fitness, and evolution of drug-resistant Mycobacterium tuberculosis. Int J Tuberc Lung Dis. 13(2009), 1456- 1466.

6. P. Brodin, L. Majlessi, L. Marsollier, M.I. de Jonge, D. Bottai, C. Demangel, O.H.J. Neyrolles, P.D.Butcher, C. Leclerc, S.T. Cole, R. Brosch,. Dissection of ESAT-6 system of Mycobacterium tuberculosis and impact on immunogenicity and virulence. Infect Immun. 74(2006), 88-98.

7. P.B.Choudhari, M.S. Bhatia, N. M. Bhatia Application of Pocket Modeling and k-nearest Neighbor Molecular Field Analysis (kNN-MFA) for Designing of some Anticoagulants: Potential Factor IXa Inhibitors. Medicinal Chemistry Research. 19(2012). 3-5.

8. R. Choure, K. S. Pitre, Structural modification of coumarin for its increased anticoagulation potency. Canadian J. Chem. Engineering \& Technology. 1(2010), 7-15.

9. S. C. Derrick, S. L. Morris. The ESAT6 protein of Mycobacterium tuberculosis induces apoptosis of macrophages by activating caspase expression. Cell Microbiol. 9(2007), 15471555 .

10. R. G. Ducati, A. Ruffino-Netto, L. A. Basso, D. S. Santos. The Resumption of Consumption - A Review on Tuberculosis. Mem Inst Oswaldo Cruz. 101(2006), 697-714.

11. M. E. Feltcher, J. T. Sullivan, M. Braunstein Protein Export Systems of Mycobacterium tuberculosis: novel target for drug development. Future Microbiol. 5(2010)1581-1597.

12. M.J. Fenton, M.W. Vermeulen. Immunopathology of tuberculosis: roles of macrophages and monocytes. Infect Immun. 64(1996), 683-690. 
13. B. B. Finlay, S. Falkow, S., Common themes in microbial pathogenicity revisited. Microbiol Mol Biol Rev. 61(1997), 136-169.

14. S. M. Fortune, A. Jaeger, D.A. Sarracino, M.R. Chase, C.M. Sassetti, D.R. Sherman, B.R Bloom, E. J. Rubin. Mutually dependent secretion of proteins required for mycobacterial virulence. Proc Natl Acad Sci. 102(2005), 10676-10681.

15. A. M. Ginsberg, A.M. Tuberculosis drug development: Progress challenges and the road ahead Tuberculosis. 90(2010) 162-167.

16. A. Jain , R. Mondal, Extensively drug-resistant tuberculosis: current challenges and threats. FEMS Immunol Med Microbiol. 53(2008), 145-150.

17. J. Keane, M. K. Balcewicz-Sablinska, H. G. Remold, G. L. Chupp, B. B. Meek, M. J. Fenton, H. Kornfeld. Infection by Mycobacterium tuberculosis promotes human alveolar macrophages apoptosis. Infect Immun. 65(1997), 298-304.

18. A.N. Leung, Pulmonary Tuberculosis: The Essentials. Radiology. 210(1999), 307-322.

19. Sugie, Y. et al. CJ-21,058, a new SecA inhibitor isolated from a fungus. J. Antibiot. (Tokyo) 55 (2002), 25-29

20. Jang, M. Y., De Jonghe, S., Segers, K., Anne, J. \& Herdewijn, P. Synthesis of novel 5amino-thiazolo[4,5-d]pyrimidines as E. coli and S. aureus SecA inhibitors. Bioorg.Med. Chem. 19(2011), 702-714

21. Tripathi, L., Kumar, P., Singh, R., Stables J. P., Design, synthesis and anticonvulsant evaluation of novel N-(4-substituted phenyl)-2-[4-(substituted) benzylidene]hydrazinecarbothioamides. European Journal of Medicinal Chemistry 47(2012),153-166.

22. Malipeddi H., Karigar AA., Malipeddi VR., Sikarwar MS., Synthesis and Antitubercular Activity of Some Novel Thiazolidinone Derivatives. TroJ of Pharm Res. 11 (2012), 61120 


\section{Figures}

Fig. 1: Synthetic scheme of designed derivatives.

Fig. 2: Hydrophobic map of Active site of SEC of Mycobacterium tuberculosis.

Fig. 3: Figure showing posed molecule M26 (Ball and Stick) with PDB ID 4UAQ.

Fig. 4: Showing the posed Molecule U8 (Ball and Stick) with PDB ID 4UAQ.

Fig. 5: Showing the posed Molecule R26 (Ball and Stick) with PDB ID 4UAQ.

Fig. 6: Showing the posed Molecule A10 (Ball and Stick) with PDB ID 4UAQ.

Fig. 7: Designed compounds with minimum inhibitory concentration (MIC). 
$\prod_{\mathrm{O}}^{\mathrm{R} 1}+\mathrm{H}_{2} \mathrm{~N}^{-\mathrm{H}} \prod_{\mathrm{S}}^{\mathrm{H}} \mathrm{NH}_{2}+\mathrm{HS} \overbrace{\mathrm{OH}}^{\mathrm{O}}$

$\downarrow \mathrm{H}_{2} \mathrm{SO}_{4}$<smiles>[R2]c1cccc(C=O)c1</smiles><smiles>[R1]c1ccc(C2SC[C@H]3SC(/N=C\C4=CCC([R20])C=C4)=NN23)cc1</smiles>

Fig. 1: Synthetic scheme of designed derivatives. 


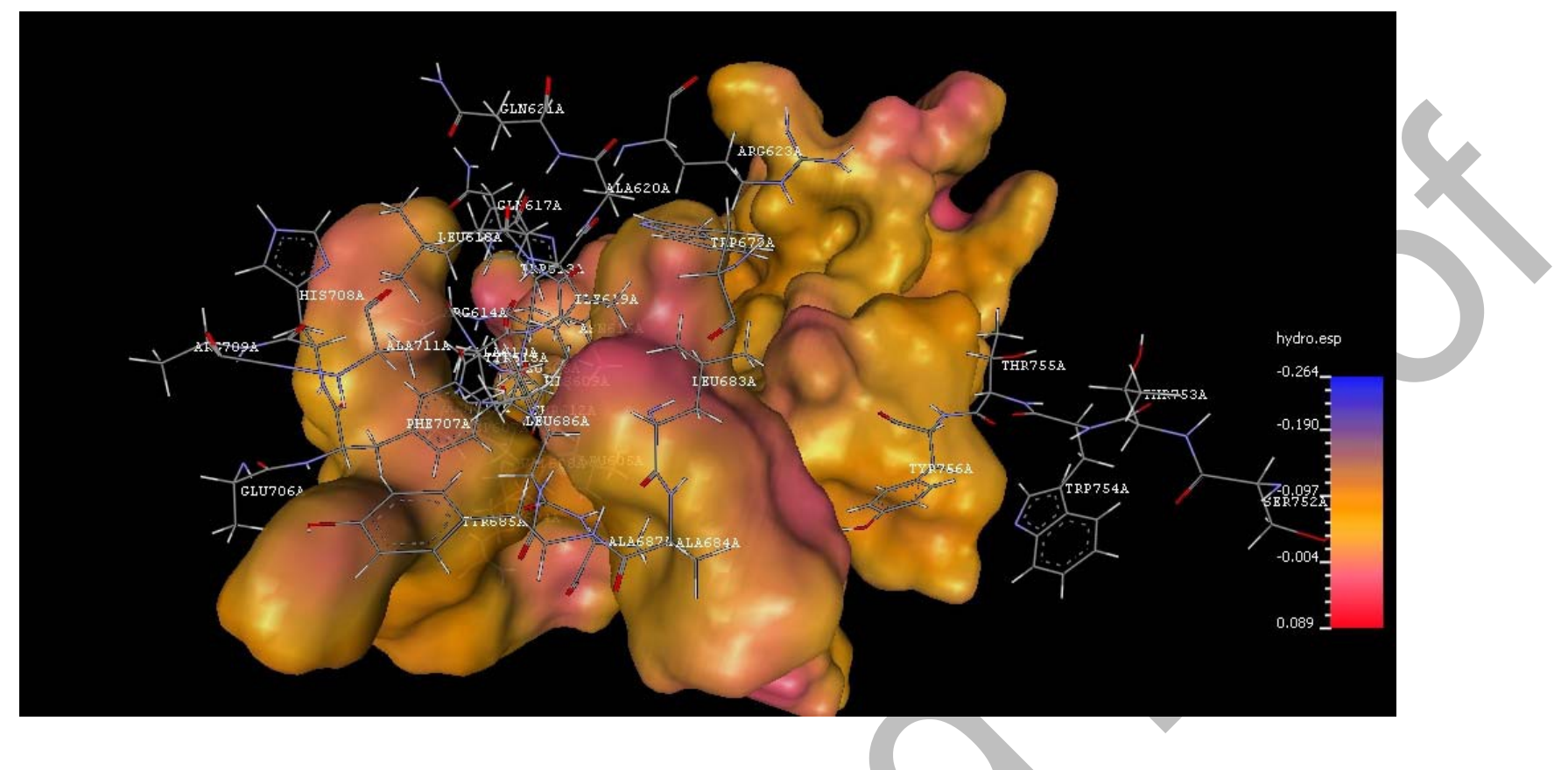

Fig. 2: Hydrophobic map of Active site of SEC of Mycobacterium tuberculosis. 

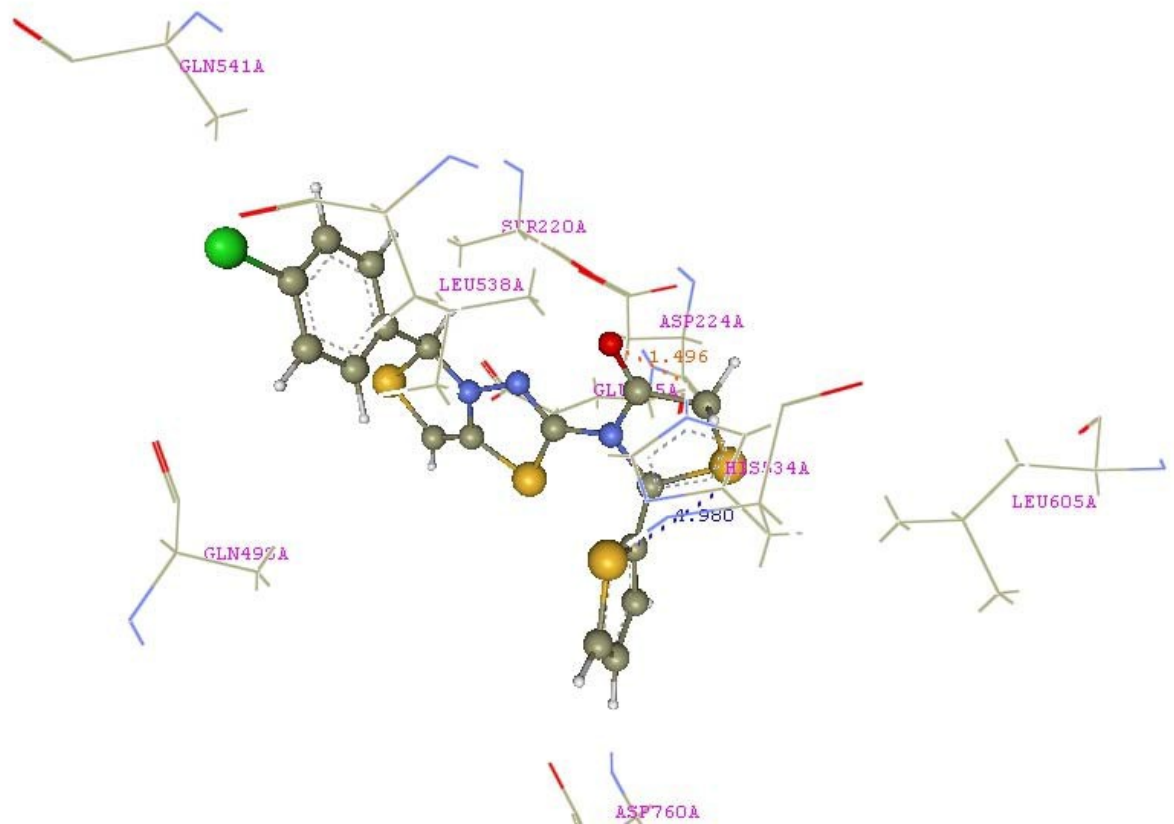

Fig. 3: Figure showing posed molecule M26 (Ball and Stick) with PDB ID 4UAQ with hydrogen bond interaction (Red Color) and Pi-Stacking Interaction (Blue Colour). 


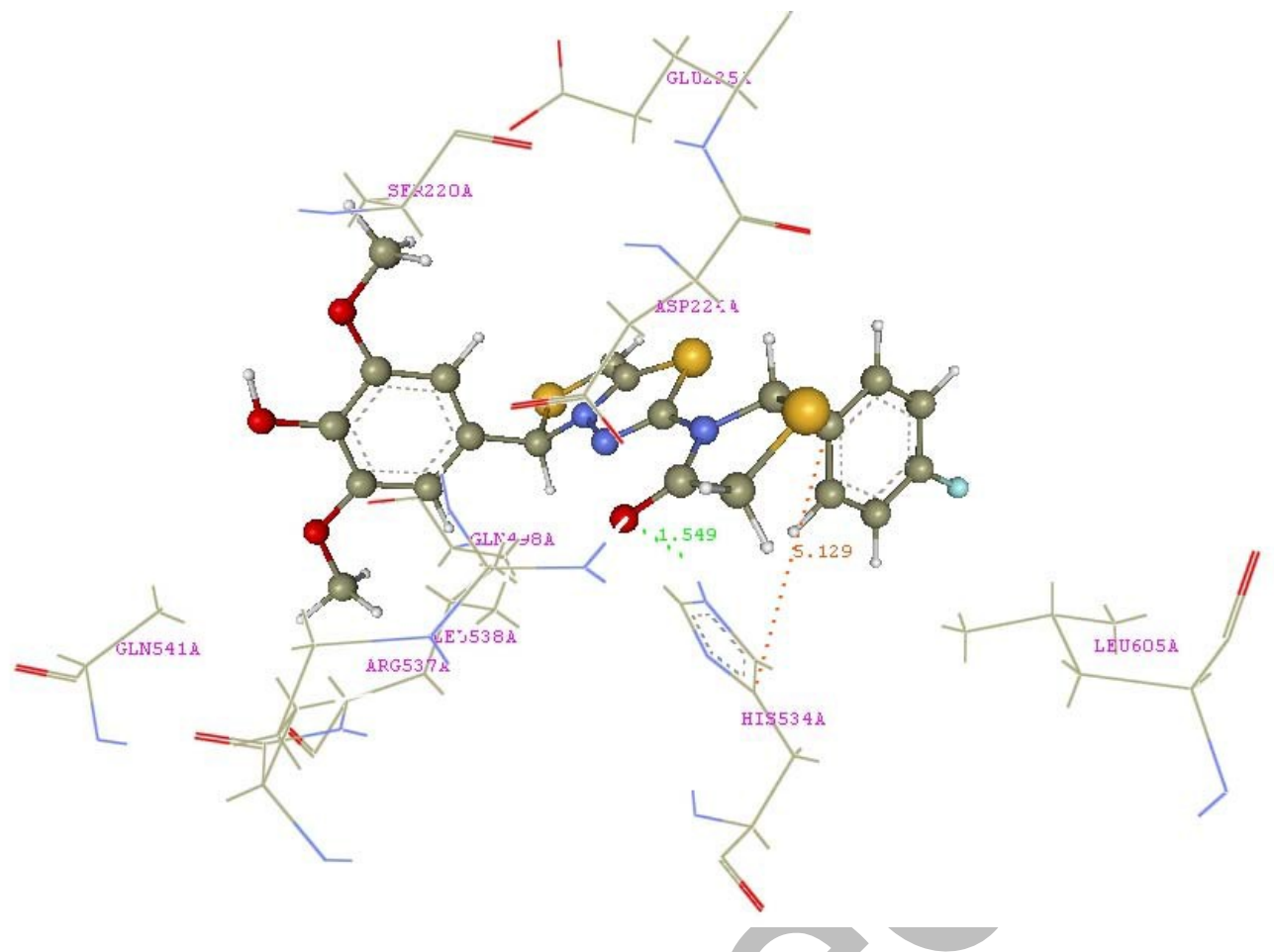

Fig. 4: Showing the posed Molecule U8 (Ball and Stick) with PDB ID 4UAQ with hydrogen bond interaction (Red Color). 


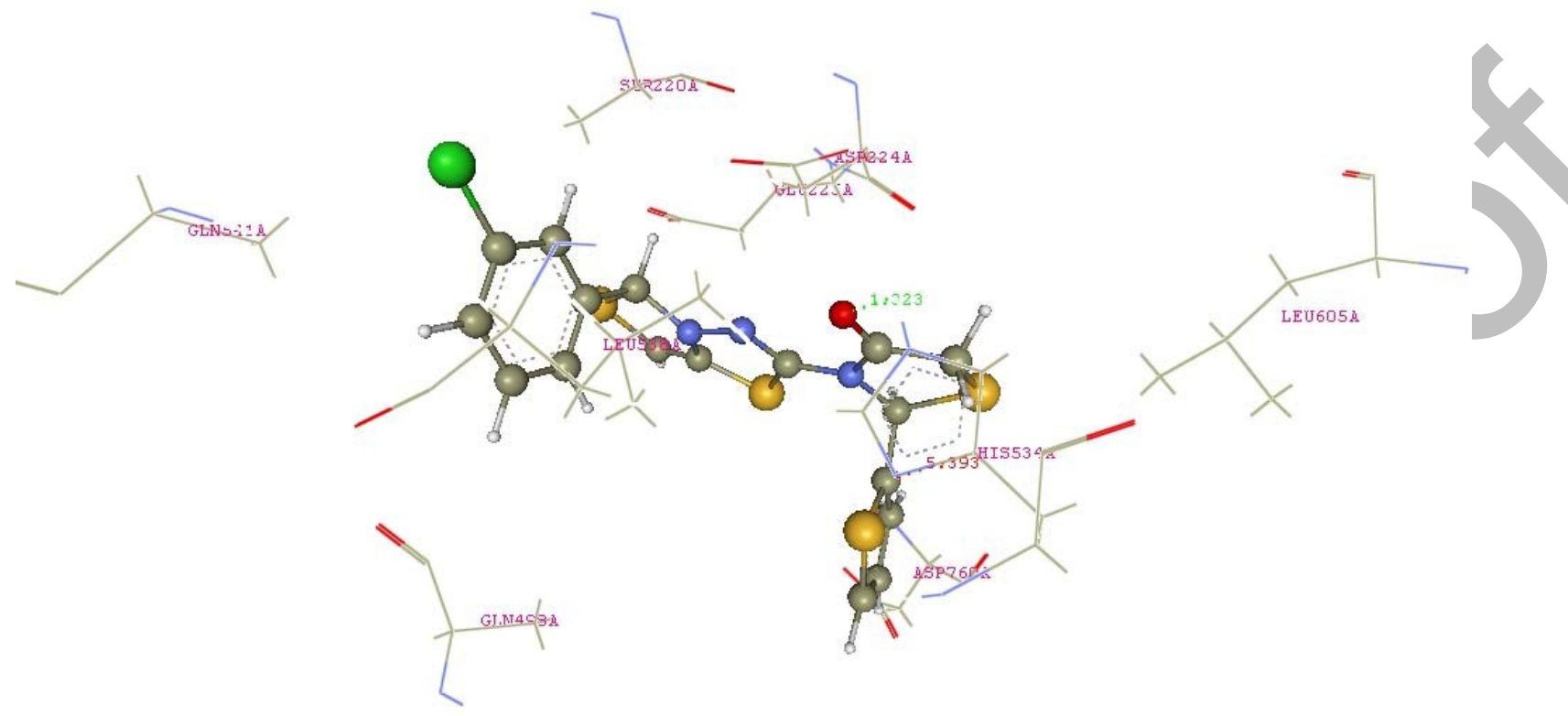

Fig. 5: Showing the posed Molecule R26 (Ball and Stick) with PDB ID 4UAQ with hydrogen bond interaction (Red Color). 


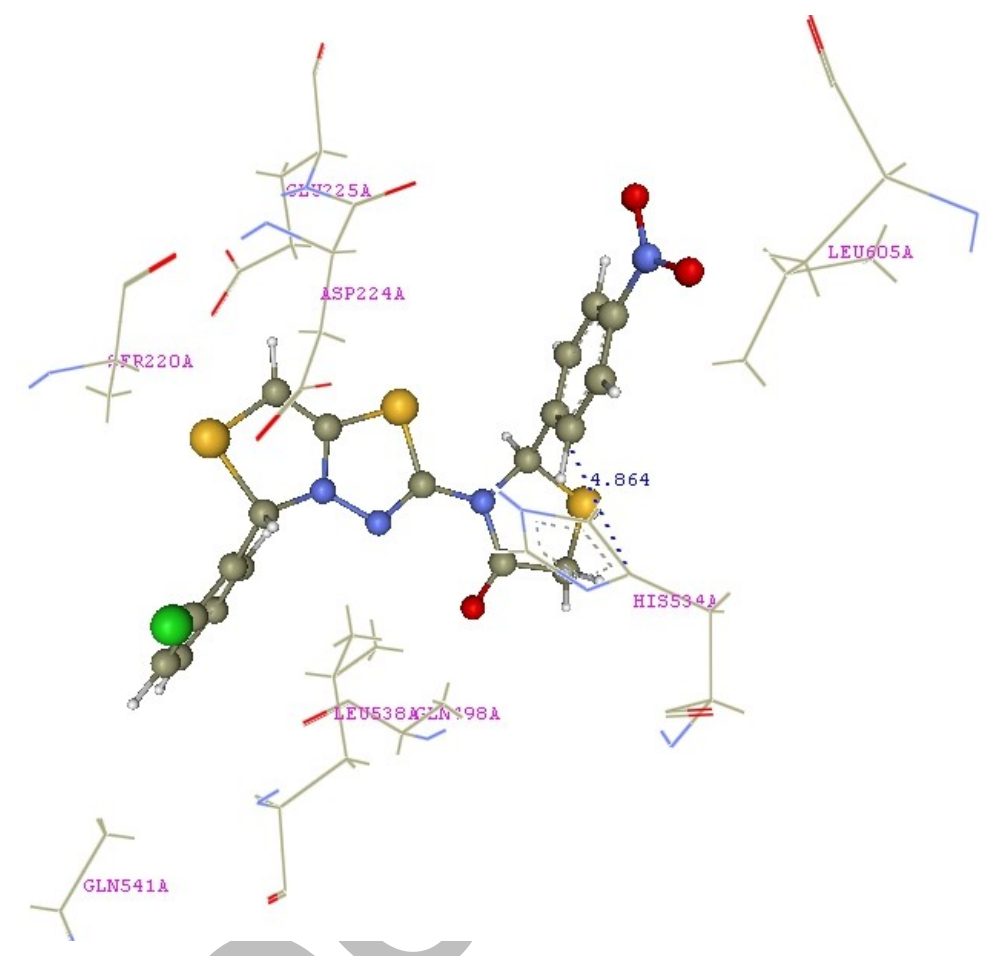

Fig. 6: Showing the posed Molecule A10 (Ball and Stick) with PDB ID 4UAQ. 


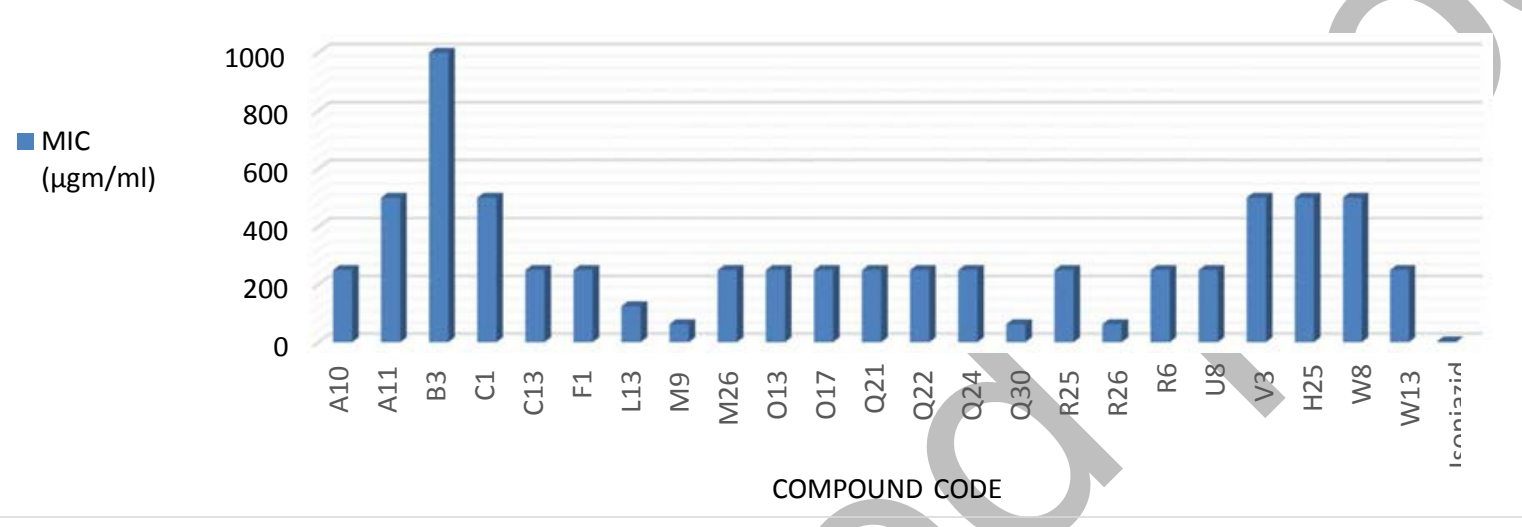

Fig. 7: Designed compounds with minimum inhibitory concentration (MIC). 